\title{
A COMPLEXITY FRAMEWORK FOR CONSENSUS AND CONFLICT
}

\author{
PETER MITIC \\ Santander UK, 2 Triton Square, Regent's Place, London NW1 3AN \\ Dept. of Computer Science, UCL, Gower Street, London WC1E 6BT \\ Laboratoire d'Excellence sur la Régulation Financière, Paris
}

\begin{abstract}
Consensus and conflict are modelled in the context of interacting pairs of agents who may have very diverging sentiments regarding some particular issue. Simulations using the model display characteristics of complexity. Agents are modelled using Beta probability density functions whose parameters determine the agent's opinion and resistance to change after an interaction, and a third independent parameter that determines the agent's influence. Interactions among groups of agents with both aligned and opposing sentiments are simulated. The results indicate that in most cases a form of consensus is reached eventually, but for opposed agents, it is not possible to tell which agents that consensus will favour. Proofs of convergence are given in the cases where the initial state is one of consensus, and when it is one of conflict.

Keywords: Beta distribution, conflict, consensus, convergence, influence, resistance, sentiment, simulation
\end{abstract}

\section{INTRODUCTION}

In this analysis we build a framework for complexity that is consistent with the characteristics of complexity stated by Rzevski in [1]: autonomy, emergence, non-equilibrium, non-linearity, connectivity, self-organisation and co-evolution. The framework, once initialised, is designed to evolve with time according to rules that are stochastically determined, and follow general principles. Those principles are consistent with Rzevski's discussion in [2], namely that a model of a complex system must be adaptable to changes, and that the adaptation must be autonomous (i.e. without specific instructions). A major consequence is that the agents in the system must be able to self-organise (see the details in Section 3).

The framework comprises a set of $n$ agents $\boldsymbol{X}=\left\{X_{1}, X_{2}, \ldots, X_{n}\right\}$. In the interaction model of Section 3, time is discretised such that times $t_{1}, t_{2}, \ldots$ are the times at which an agent-agent interaction occurs. The resulting time intervals, $t_{2}-t_{1}, t_{3}-t_{2}, \ldots$ are therefore not necessarily of constant length. The framework is used to examine the general principles of conflict and consensus in the context of agreement or disagreement on some issue. We say that an agent has a particular sentiment with respect to an issue, and measure that sentiment by a real number in $(0,1)$ which represents the degree to which the agent either agrees or disagrees with some proposition.

\subsection{Conflict and Consensus}

Any pair of agents can "agree" or "disagree" with each other with respect to an issue. Agreements constitute consensus, and large disagreements constitute conflict. The precise definition of an agent (Section 3.1) will clarify the distinction between conflict and consensus adequately. The development over time of opinions of a group of agents will be simulated (Section 4). The complexity-derived simulations determine whether or not consensus can be reached following initial conflict, and whether or not conflict can arise from initial consensus. 
The interaction between agents is modelled by the balance of influence of one agent and resistance of another. That balance results in emergent behaviour: non-deterministic results which sometimes cannot be explained precisely.

\section{PREVIOUS WORK ON CROWDS, CONFLICT AND CONSENSUS}

This section contains a brief account of studies of agent interaction that relate to consensus or conflict. Early work in this context was done by Epstein [3], and it forms the basis of many subsequent studies. Epstein states principles, broadly the same as the ones stated in the previous section, in the form of interaction rules. However, no specific model is proposed, and emergence is not mentioned. In a later paper [4], Epstein introduces a rule-based model in which the state of a non-authority set of agents can be changed by an authority set of agents. The model incorporates hard thresholds: breaching a threshold results in a state change.

Epstein's principles persist in the review of Lemos et al. [5], with the addition of emergence. The context remains social conflict. They discuss, first, threshold-based models, such as from Granovetter [6]. Each agent has a threshold, and joins a protest if the number of other agents in the protest exceeds the threshold. Among other more specialised models cited are papers by Doran [7] (weak but agile agents against strong but sluggish agents), and Kim \& Hanneman [8] (rules for opposing agents are distribution-based). In the context of threshold models, we also mention Gurr's Relative Deprivation model [9], in which an agent's increasing disparity between expectation and reality leads to a change of state.

Game theory is a second, and common alternative, approach to agent interaction. This choice is natural, since such models use constraints on actions due to other agents. Jones [10] gives a comprehensive overview of conflict and consensus models with non-cooperative and cooperative game theory respectively. Among the concepts considered are threats, bargaining and strategy. Axtell et al. [11] models agent interaction as a game where two players bargain for ownership of a resource. If their joint demand exceeds some predetermined limit, they both get nothing. Otherwise, they get their demands. All possible outcomes are shown, as is the location of each agent with respect to those outcomes. Weisbuch [12] extends the Axtell analysis to include features (e.g. a moving average of previous gains) for each agent. Weisbuch's result shows that there is a stabilisation as time increases, and that divergence does not emerge spontaneously from a random starting point, agreeing with results in Section 4 of this paper.

Game theory models tied to a network represent a third mode of agent interaction. Many have the Voter model [13] as a basis. Within a network, a target agent having a discrete set of opinions is selected at random, and is then assigned the opinion of one of its nearest neighbours, also chosen at random. In terms of the network topology context, the 'nearest' neighbours are the agents that are directly connected to the target agent. Physical distance between the target agent and any other agents is not relevant: network connections are virtual.

This opinion-formation mechanism is best suited as a model of agents' lack of self-confidence, resulting in imitation. Under assumptions that state transitions are binary (only two opinions, with a potential transition of one to another), and that such transitions follow a Markov process, agents' opinions converge to one of the possible opinions. The Axelrod model [14] is an extension of the Voter model in that each agent is assigned a set of features. In an agent-agent interaction, corresponding features are compared, and if they differ, the features of one agent are set to the features of the other. After multiple interactions, the Axelrod model predicts either global convergence, or the emergence of several groups. The Axelrod model is appropriate when applied to social groups that have multiple distinct characteristics, some of which are opposed. Simulations and summaries for both the Voter and Axelrod models are shown in Miguel et al. [15]. 


\section{AGENTS AND AGENT INTERACTION}

In this section we describe a new way to represent agents and to investigate interactions between them. This analysis differs from the prior analyses (Section 2) in that the context in which the agents interact is completely general. No assumptions are made apart from the general principles underlying complexity. The Mathematica implementation permits, a suitable combination of mathematical formulation, manipulation ability, and display. Example applications are given in this section, and in Section 4.

\subsection{Agent Structure}

An agent $A$ is represented by a triple:

$$
A=\{(a, b), I, s\} .
$$

In (1), $a$ and $b$ are the parameters of a Beta density $\beta(a, b, x) ; x, a, b \in(0,1), I \in(0,1)$ represents the agent's influence and $s$ is an alphanumeric name for the agent. With those values of $a$ and $b$, a Beta density has a single peak with a skew that depends on the values of $a$ and $b$. The degree to which an agent agrees or disagrees with an issue (i.e. the agent's opinion) is modelled by the location of the peak of the density in $(0,1)$. Specifically, an agent's location $L(A)$ is given by the mean of the density,

$$
L(A)=\frac{a}{a+b}
$$

An agent with a biased opinion is represented by either $a<b$ (the peak is near $x=0$ ) or $b<a$ (the peak is near $x=1$ ). The parameter values in very biased cases are approximately in the ratios 1:10 and 10:1 respectively. An agent with a neutral opinion has $a=b$, and that opinion is most open to influence if $a$ and $b$ are small (typically less than 10). Both very biased and near neutral opinions are typically found in political contexts. Most recently very biased opinions have been noted with respect to referenda. Examples are Brexit, Scottish independence and Catalonian independence. In the illustrations in the following section, an agent with very low influence $(I \sim 0)$ is shown with very low opacity, and an agent with very high influence $(I \sim 1)$ is shown with very high opacity. Intermediate opacities indicate medium influence $(I \sim 0.5)$. It is easy to identify people who are or have been influential. The result of their influence may only be apparent with hindsight. One example is Rosa Parks, who triggered the campaign for racial equality in the USA in 1955. Another is Nelson Mandela, who had a key role in formulating proposals to end apartheid in South Africa in 1989.

\subsection{The Mechanism for Agent Interaction}

The way in which agents interact is a more sophisticated version of the mechanism used by Burt [16]. The mechanism uses an agent's resistance to influence, $\rho$, which is defined in terms of the variance, $v$, of the agent's underlying Beta $(a, b)$ distribution, ultimately mapped to $(0,1)$ using a sigmoid transformation on the inverse square root of $v\left(\rho^{\prime}\right.$ in $(3)$, below).

$$
v=\frac{a b}{(a+b)^{2}(a+b+1)} ; \rho^{\prime}=\frac{1}{\sqrt{v}} ; \rho=\frac{2}{1+e^{-\frac{\rho^{\prime}}{25}}}-1
$$


When two agents $X\left(\left(a_{X}, b_{X}\right), I_{X}\right.$, , $X$ "') and $Y\left(\left(a_{Y}, b_{Y}\right), I_{Y}\right.$, ,'Y'”) interact, the convention used is that $Y$ affects $X, Y$ remains unchanged afterwards, and $X$ transforms to a resultant, $Z\left(\left(a_{Z}, b_{Z}\right)\right.$, $I_{Z}$, "Z"), denoted by $Z=\langle X, Y\rangle$. The interaction proceeds by defining Beta parameters $\left(a_{Z}\right.$, $b_{Z}$ ) for $Z$ according to Algorithm 1, below.

Algorithm 1: Interaction $Z=\langle X, Y>$

Step 1: Calculate a linear combination of influences.

$$
\begin{gathered}
r_{1}=\frac{2 I_{Y}}{I_{Y}+\rho_{Y}}-1 ; r=\frac{1}{1+e^{-2 r_{1}}} ; \\
a_{Z}^{\prime}=r a_{X}+(1-r) a_{Y} ; b_{Z}^{\prime}=r b_{X}+(1-r) b_{Y}
\end{gathered}
$$

Step 2: Modify the Beta parameters in Step 1 based on resistances, with a bias towards $X$.

$$
a_{Z}=\sqrt{\rho_{X}} a_{X}+\left(1-\sqrt{\rho_{X}}\right) a_{Z}^{\prime} ; b_{Z}=\sqrt{\rho_{X}} b_{X}+\left(1-\sqrt{\rho_{X}}\right) b_{Z}^{\prime}
$$

Step 3: Modify the Beta parameters in Step 2 by perturbations (see the explanation below). Using single draws $\varepsilon_{a_{z}}$ and $\varepsilon_{b_{z}}$ from $N\left(0, a_{z} / 10\right)$ and $N\left(0, b_{z} / 10\right)$ distributions respectively,

$$
a_{z} \rightarrow a_{z}+\varepsilon_{a_{z}} ; b_{z} \rightarrow b_{z}+\varepsilon_{b_{z}} .
$$

Step 4: Use a similar stochastic perturbation to derive the influence of $Z$, where $\varepsilon_{I_{X}}$ is a single draw from a $N\left(0, I_{X} / 100\right)$ distribution.

$$
I_{Z}=I_{X}+\varepsilon_{I_{X}}
$$

Step 5: Apply limiters such that the results of steps 3 and 4 are in $(0,1)$

The perturbation stages ( 3 and 4 ) add a non-linear element to the Beta parameter transformations. They make the interaction process non-deterministic, and model the situation where agents do not make entirely rational or expected decisions. An example of such a decision is the election of Donald Trump as US President. Overall, the effect of the Algorithm 1 rules say: "Balance influence and resistance with a bias towards the opinion of the influenced agent." Figures $1 \mathrm{a}$ and $\mathrm{b}$ show two contrasting interactions using the Algorithm 1 rules. They both

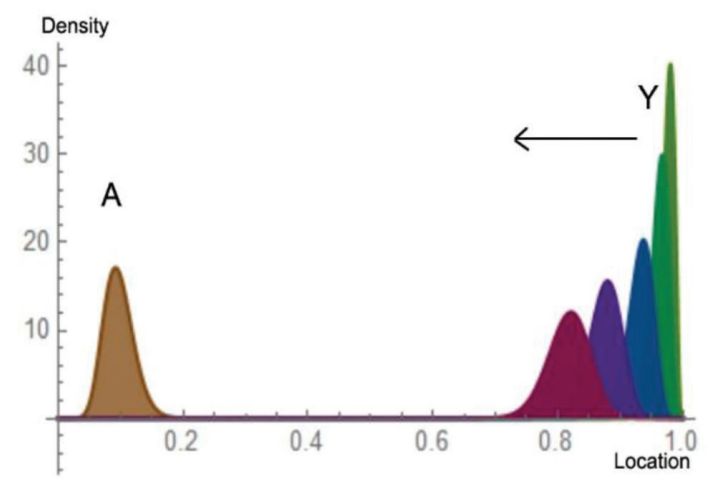

Figure 1(a): Influential $A$ has a has a limited effect on resistant $Y$ 


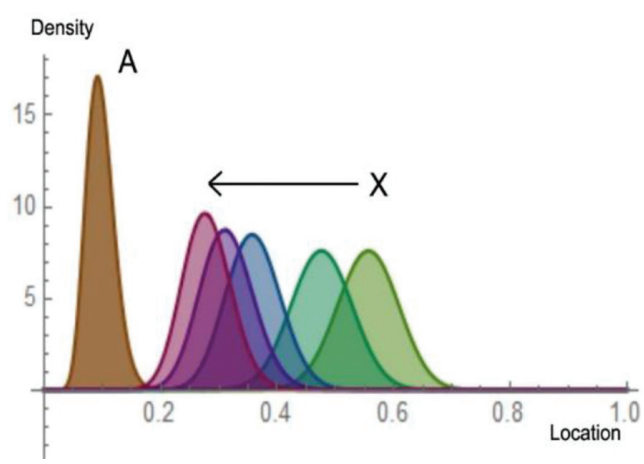

Figure 1(b): Influential $A$ significant effect on non-resistant $Y$.

illustrate the repeated effect of an agent $A$ a second agent. In Fig. 1a, the resultants of successive interactions are given by $Y_{r+1}=Y_{r}, A, r=1 . .4 ; Y_{1}=Y$. In this case, $A$ is a very influential (high opacity) agent with high resistance, who tries to influence a $Y$ who is equally influential and resistant, but whose opinion directly opposes $A$ 's opinion. $A$ has some success in shifting $Y$ 's opinion, as shown by the gradual progression of $Y$ from location near 1 to location near 0.8 (the left-pointing arrow). This is a model of, for example, unsuccessful advertising. In Fig. 1b, $Y$ is replaced by an agent $X$ who has medium influence (shown as lower opacity), has lower resistance to persuasion, and is not opinionated. $A$ is able to shift $X$ 's opinion significantly to a location near 0.3 . Successive resultants in this case are given by $X_{r+1}=X_{r}, A, r=1 . .4 ; X_{1}=X$. This situation models successful advertising, and also successful transmission of influence by social media.

\subsection{Group Location and Convergence}

A Group is a set of agents. The Group Location is a weighted average of the locations of the members of a Group. The weights are normalised ratios of influence to resistance for each member of the Group (Equation 4). So for a Group $X$ with $n$ agents $\left\{X_{1}, X_{2}, \ldots, X_{n}\right\}$,

$$
w_{i}=\frac{I\left(X_{i}\right)}{\rho\left(X_{i}\right)}, i=1 . . n ; L(\boldsymbol{X})=\sum_{i=1}^{n} w_{i} L\left(X_{i}\right) .
$$

Following a sequence of agent interactions among members of a Group, the Group Location eventually converges to a consensus. The term 'convergence' is used in the sense that there exists a confidence interval in which the Group Location will lie following all interactions. Appendix A is a proof that, under certain conditions, the Group Location for the case $n=2$ is bounded by a subset of $(0,1)$. Appendix B extends this result to the case $n>2$. In the case of agents whose opinions broadly agree, the eventual opinion will also broadly agree with the initial opinions. If two subsets are markedly opposed, there will be consensus eventually, but it is not possible to say which subset will be favoured, despite a bias towards influenced agents who are resistant to change (Step 2 in Algorithm 1).

\section{SIMULATION RESULTS}

The principles introduced in the previous section are illustrated in this section. The appendices give proofs that predict what the results of simulations should be, and the simulations are consistent with those predictions. 
Consider $r$ interactions for the Group $\boldsymbol{X}$ of Section 3.3. At each interaction pairs of agents are chosen at random, with replacement. If the pair comprises two identical agents, the interpretation is that the agent changes its opinion independently of other agents. The emergent behaviour is then examined by generating pair-wise interactions repeatedly. The existence of an implied fully connected network with agents as nodes is assumed. Notable cases of influence and resistance follow. In each case, the plots presented show the Group Location directly after each interaction. In each case $n=10$ and $r \geq 200$. Note that the interval between successive interactions is not necessarily constant. In each simulation, when agent $X_{p} \in X$ tries to influence agent $X_{q} \in X$, let the resultant after the $r^{\text {th }}$ interaction of $N$ for $X_{q}$ be $X_{[N, q, r]}$. Then $X_{[N, q, r+1]}$ is defined by Equation 5a. Equation 5 b gives the Group Location $L\left(\boldsymbol{X}_{r+1}\right)$ after $r$ interactions (the weights $w_{i}$ are defined in Equation 4).

$$
\begin{gathered}
X_{[N, q, r+1]}=\left\langle X_{[N, q, r]}, X_{p}\right\rangle, r=1 . . N ; X_{[N, q, 1]}=X_{q} \\
L\left(X_{r+1}\right)=\sum_{i=1}^{N} w_{i} L\left(X_{i, r+1}\right)
\end{gathered}
$$

The Group Locations are illustrated in the figures that follow. If $p=q$, the interpretation is that $X_{q}$ has influenced itself (i.e. has had a change of mind).

\subsection{Consensus: Distinct Groups, each with Similar Opinions}

Figure 2(a) shows three distinct Groups $G 1, G 2$ and $G 3$ in their initial states, and Fig. 2b shows them after 200 repeated interactions. The 'before' and 'after' states look very similar. $G 1$ and $G 3$ are highly opinionated and remain clustered around their respective start locations, 0.9 and 0.1. G2 has no firm opinion and remains clustered near its starting location, 0.5. Figure 2(c) shows the corresponding Group Location for each Group. The G1 and G3 Group Locations are bounded within narrow limits, whereas $G 2$ exhibits greater volatility (see Appendix A). These results reflect greater susceptibility of $G 2$ to influence. These cases are typical of political groupings: some people are highly opinionated with respect to certain political parties, and they mostly stay that way. Others are willing to be swayed.

The results obtained in this simulation resemble those obtained by Namatame and Chen [17] in the context of reinforcement learning. They derive similar-shaped probability distributions in simulations, but have no explicit densities.

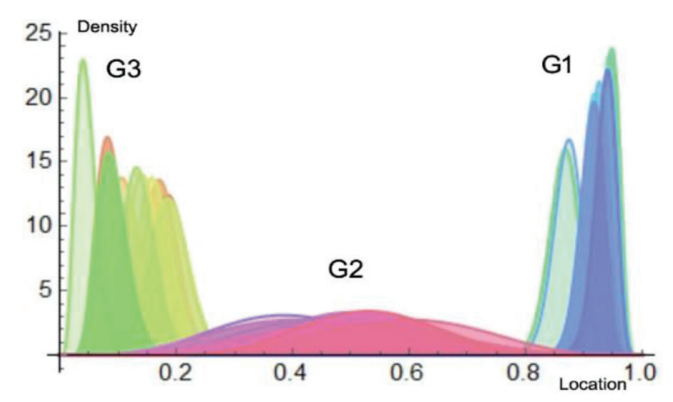

Figure 2(a): Three Groups initial state 


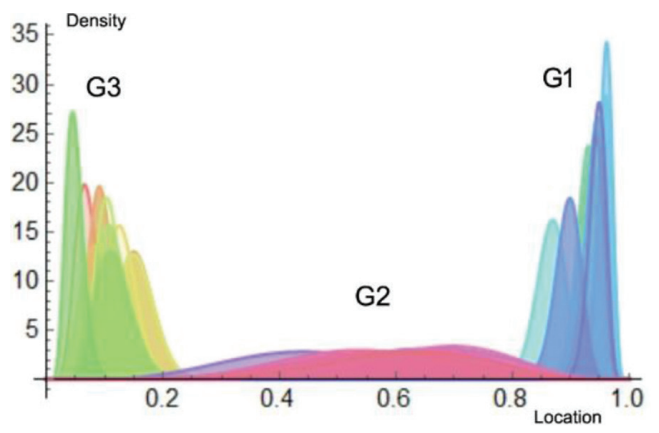

Figure 2(b): Three Groups final state

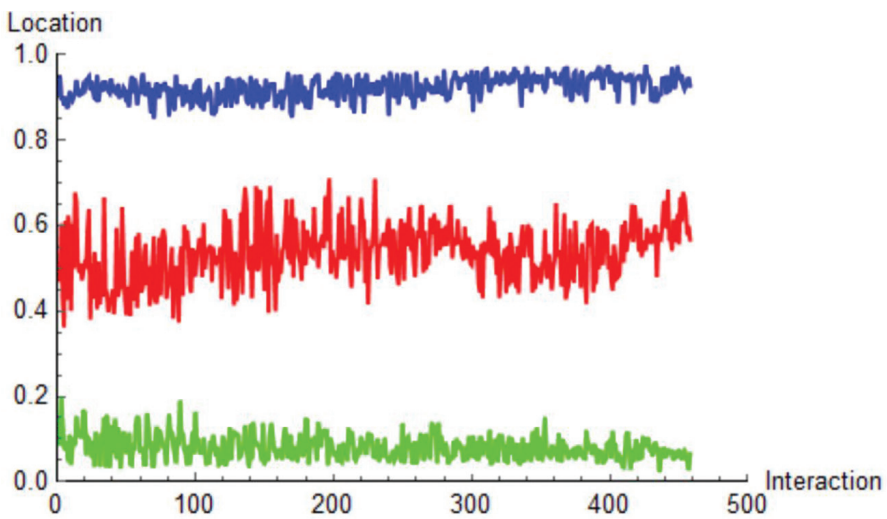

Figure 2(c): Three Groups: Time progression of Group Location (G3 in green, $\mathrm{G} 2$ in red, G1 in blue)

\subsection{Conflict: Groups with Opposing Opinions}

Figure 3 shows the progressive Group Locations of three distinct simulations for the same two agents, who have directly opposing strong opinions, as shown by their initial locations: 0.95 and 0.05 . The simulations have common characteristics: initial volatility followed by local convergence (as predicted in Appendix B). However, the limit points are different for every simulation. If a simulation is run for 1000 interactions, the pattern of shifting the Group Location of the pair between the limits 0 and 1 continues. There is an eventual 'winner' of the argument, but it is not possible to tell in advance which agent will be the winner. This result is really the only one of the results presented here that is surprising. It is not clear, in advance of running a simulation, that any such consensus might be reached. A plausible expected result is continued conflict. The Anglo-Irish Good Friday agreement of April 1998 provides an example of where two conflicting parties eventually agreed on the issue of peace in Northern Ireland. Prior to that date it was not clear if an agreement would favour one side or the other, or to what extent.

\subsection{The Effect of one Influential Agent on a Group}

The illustration in this section (Fig. 4) shows the effect of a single influential agent, who joins a Group after the original Group has been interacting for some time. The entry point is at 


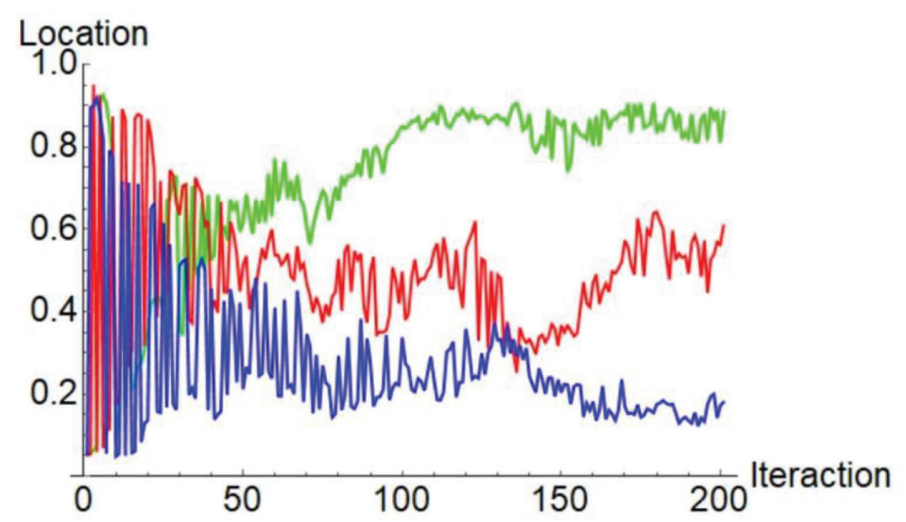

Figure 3: Three simulations: two opposed agents

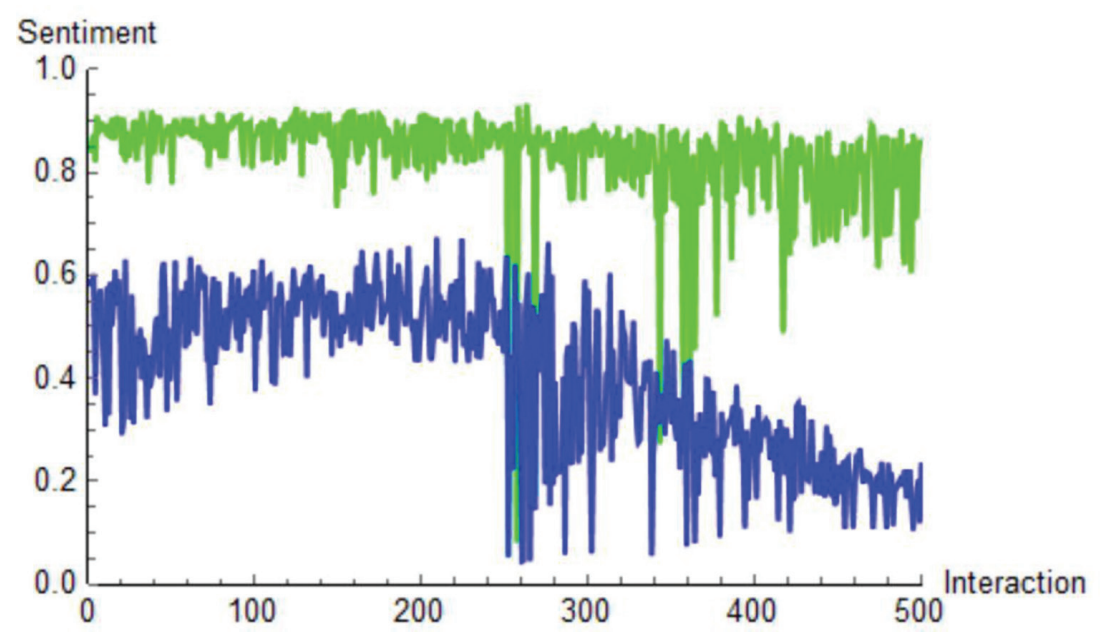

Figure 4: An opposing agent, entering at Interaction 250, having limited influence on a very biased Group (green trace), but more influence on a neutral Group (blue trace)

Interaction $=250$ : the half-way stage. Two traces are shown. The first represents a Group that holds a strong biased opinion, and the second represents a Group that holds an overall neutral opinion. The agent that enters has a strong opinion that opposes the first Group, and induces a period of volatility in the Group Location of both Groups.

If the Group has a hardened initial opinion, the opposing agent cannot really influence its members significantly. The dips in the Group Location show that the opposing agent has some effect, but there is no trend towards the opposing opinion for the original Group. As time progresses, the opposing agent is only able to generate volatility. Alternatively, the influencer can influence permanently the Group that was neutral initially. For such a Group, the Group Location trace moves towards the influencer's location, and stays there. There is inherently more volatility for a Group that is neutral initially. In both cases volatility increases markedly when the influencing agent enters. This simulation illustrates two points. First, the effect of a single influential agent on a Group can be significant. An analysis of the Brexit referendum (https:// 
www.referendumanalysis.eu/eu-referendum-analysis-2016/section-7-social-media/impact-ofsocial-media-on-the-outcome-of-the-eu-referendum/) indicates that the Vote Leave campaign had a significant effect on the outcome. Second, conflict can arise where there was initial consensus. Brexit provides an example here too: a rise in hate crime was widely reported following the referendum (https://www.independent.co.uk/news/uk/home-news/racist-hate-crimessurge-to-record-high-after-brexit-vote-new-figures-reveal-a7829551.html).

\subsection{Random Interactions with varying Group size}

Two sets of randomly-defined agents (with 10 and 100 agents respectively) interact separately. In both cases their influences span the range $(0,1)$ and their Beta distribution parameters are randomly chosen integers from the set 2..202. This allows for a comprehensive range of resistances and biases. We observe a much greater variation in the Group Location for the "100 agent" case (Fig. 5). Similar simulations show that as the Group size increases, so does the variation in the Group Location. The conclusion is that the more agents there are in the Group, the less likely it is that consensus will be reached. Large corporate organisations are examples where consensus is slow to emerge, whereas small startups are usually much faster. (https://www.fastcompany.com/3049164/how-to-make-decisions-more-efficiently).

\section{DISCUSSION AND SUMMARY}

The simulation results in Section 4 indicate that agents who 'agree' initially, continue to 'agree' (Section 4.1), a conclusion which makes intuitive sense. If agents 'disagree' initially (Section 4.2), the outcome is not decidable in advance, but converges to some subset of the range $(0,1)$. Consequently, the outcome may favour one or other of the opposing agent Groups more than the other, or possibly neither. In one sense the outcome is therefore highly unsatisfactory because there will always be one aggrieved party. Yet the existence of some outcome rather than none can be considered an advance. In some cases any tolerable outcome is highly desirable, such as peace negotiations that lead to an end to physical violence. An example is the eventual resolution of the Irish "troubles" of the latter half of the 20th century with the Good Friday agreement in April 1998. The possibility still exists that no consensus will be reached, and some simulations show that. In practical terms, it can be argued that consensus must emerge eventually, since continued conflict eventually becomes intolerable to people involved. The Arab-Israel dispute is an example of a long-running dispute which appears to have been intractable since the 1917 Balfour Declaration, which called for the establishment

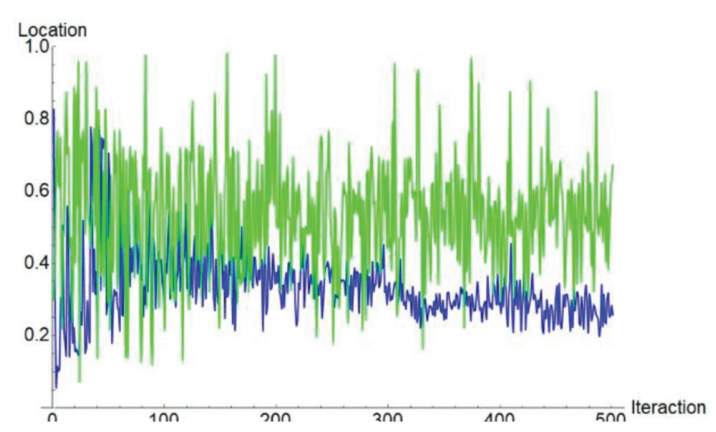

Figure 5: Random Interactions: Group size 10 (blue trace) and 100 (green trace) 
of a homeland for the Jews in Palestine. Even that dispute has had its quieter moments (i.e. a tacit consensus), although conflict continues to flare up.

The conclusion that a single agent can have a fast significant effect on undecided agents (Sections 4.3) is noteworthy in the context of advertising or political campaigns. Arguably, influential figures were a major deciding factor in the 2016 Brexit referendum. However, the simulations of Section 4.4 show that an influential agent is unlikely to change the opinion of agents who are directly and strongly opposed in opinion. Section 4.5 illustrates a result that makes any prospect of consensus difficult: the more agents there are, the harder it is to agree.

As a suggestion for further work, we propose a change to deal with an unsatisfactory situation concerning the adequacy of the description of a Group comprising two distinct partitions, each with opposing opinions. In Section 3.3, the location of such a Group, or any other Group, is given as a weighted average of the locations of the members of the Group. This is problematic because it effectively describes an agent whose location is midway between two extremes: a situation that does not exist. A possible way out is to adopt the concept of a superposition of two opposing locations, which is essentially a quantum approach. The Group Location of a pair of agents would then be simultaneously two values, to be resolved on observation.

\section{REFERENCES}

[1] Rzevski, G. \& Skobelev, P., Managing Complexity. WIT Press: Boston, 2014.

[2] Rzevski, G., A practical methodology for managing complexity. Emergence: Complexity \& Organization, 13(1/2), pp. 38-56, 2011.

[3] Epstein, J.M., Agent-based computational models and generative social science. Complexity, 4(5), pp. 41-60. 1999. https://doi.org/10.1002/(sici)1099-0526(199905/06)4:5<41::aid-cplx9>3.3.co;2-6

[4] Epstein, J.M., Modeling civil violence: an agent-based computational approach. Proceedings of the National Academy of Sciences of the USA, 99, pp. 7243-7250, 2002. https://doi.org/10.1073/pnas.092080199

[5] Lemos, C., Coelho, H. \& Lopes, R.J., Agent-based modeling of social conflict, civil violence and revolution: state-of-the-art-Review and further prospects. European Conference on Multi-Agent Systems (EUMAS), pp. 124-138, 2013.

[6] Granovetter, M., Threshold models of collective behavior. American Journal of Sociology, 3(6), pp. 1420-1443, 1978.

https://doi.org/10.1086/226707

[7] Doran, J. Iruba: An Agent-Based Model of the Guerrilla War Process. Proceedings 3rd Conference of the European Social Simulation Association, Koblenz, pp. 198-205, 2005.

[8] Kim, J.W. \& Hanneman, R.A., A computational model of worker protest. Journal of Artificial Societies and Social Simulation, 14(3), 2011. https://doi.org/10.18564/jasss. 1770

[9] Gurr, T.R., Why Men Rebel, Princeton, 1970.

[10] Jones, A.J., Game Theory: Mathematical Models of Conflict. Horwood Publishing: West Sussex, 2000.

[11] Axtell, R.L., Epstein, J.M. \& Young, H.P., The Emergence of Classes in a Multi-Agent Bargaining Model. Social Dynamics, eds. S.N. Durlauf \& H.P. Young), MIT Press: West Sussex, 2001. 
[10] Weisbuch, G., Persistence of discrimination: Revisiting Axtell, Epstein and Young, available at https://arxiv.org/pdf/1706.02573.pdf, 2017

[11] Liggett, T.M., Interacting Particle Systems, Springer, 1985

[12] Axelrod, R., Dissemination of culture: a model with local convergence and global polarization. Journal of Conflict Resolution, 41, pp. 203-226, 1997. https://doi.org/10.1177/0022002797041002001

[13] Miguel, M.S., Eguiluz, V.M., Toral, R. \& Klemm, K., Binary and multivariate stochastic models of consensus formation. Computing in Science \& Engineering, 7(5), pp. 67-73, 2005. https://doi.org/10.1109/mcse.2005.114

[14] Burt, G. Conflict, Complexity and Mathematical Social Science, Vol. 15, Emerald Group, 2010.

[15] Namatame, A. \& Chen, S-H., Agent-based Modelling and Network Dynamics. Oxford University Press: Oxford, 2016.

\section{APPENDIX A: BOUNDS ON GROUP INTERACTIONS OF AGENTS}

This result is first mentioned in section 3.3. The resultant of the interaction between two agents $X$ and $Y, Z=\langle X, Y\rangle$, has location $L_{Z}$ bounded by (the notation is explained below):

$$
\max \left(0, \frac{a_{X}\left(1-\frac{s a_{X}}{10}\right)}{a_{Y}\left(1+\frac{s a_{Y}}{10}\right)+b_{Y}\left(1+\frac{s b_{Y}}{10}\right)}\right)<L_{Z}<\min \left(1, \frac{a_{Y}\left(1+\frac{s a_{Y}}{10}\right)}{a_{X}\left(1-\frac{s a_{X}}{10}\right)+b_{X}\left(1-\frac{s b_{X}}{10}\right)}\right) .
$$

Further, this result can be extended to multiple interactions between agents.

This result is proved by first considering two agents. Let agents $X$ and $Y$ have respective locations $\left\{L_{X}, L_{Y}\right\}$, and let their Beta distribution parameters be $\left\{a_{X}, b_{X}\right\}$ and $\left\{a_{Y}, b_{Y}\right\}$ respectively. Consider the first two steps of Algorithm 1 . If $I \in(0,1)$ and $R \in(0,1)$ are factors representing influence and resistance respectively (see Section 3.2), and $a^{\prime}$ is an intermediate parameter, then, supposing $a_{X}<a_{Y}$ (without loss of generality):

$a^{\prime}=I a_{X}+(1-I) a_{y}($ Algorithm 1, step 1), and

$a_{Z}=R a_{X}+(1-R) a^{\prime}$ (Algorithm 1, step 2).

$$
\begin{gathered}
\therefore a_{Z}=(I+R-I R) a_{X}+(1-(I+R-I R)) a_{Y} \\
\therefore a_{X}<a_{Z}<a_{Y} \text { and similarly } b_{X}<b_{Z}<b_{Y} . \\
\therefore a_{X}+b_{X}<a_{Z}+b_{Z}<a_{Y}+b_{Y} .
\end{gathered}
$$

From (A2 and A1),

$$
\left(\frac{a_{X}}{a_{Y}+b_{Y}}\right)\left(\frac{a_{X}+b_{X}}{a_{Y}+b_{Y}}\right)<\frac{a_{Z}}{a_{Z}+b_{Z}}<\left(\frac{a_{Y}}{a_{Y}+b_{Y}}\right)\left(\frac{a_{Y}+b_{Y}}{a_{X}+b_{X}}\right)
$$




$$
\therefore L_{X}\left(\frac{a_{X}+b_{X}}{a_{Y}+b_{Y}}\right)<L_{Z}<L_{Y}\left(\frac{a_{Y}+b_{Y}}{a_{X}+b_{X}}\right) \text {. }
$$

Note that if $a_{X}+b_{X}>a_{Y}+b_{Y}$, equation (A3) reduces to $L_{X}<L_{Z}<L_{Y}$. In the subsequent perturbation stage, the limits set in (A3) are extended outwards accordingly. The standard error $s$ corresponds to a given confidence level on a Normal density $N\left(0, \frac{c}{10}\right)$, where $c$ is a placeholder for $a_{X}, b_{X}, a_{Y}$ or $b_{Y}$. For example, at $95 \%$ confidence, the 2-tailed value of $s$ is 1.96. The upper limit in (A3) is then increased to a $95 \%$ confidence maximum:

$$
L_{Y}\left(\frac{a_{Y}+b_{Y}}{a_{X}+b_{X}}\right) \rightarrow \frac{a_{Y}\left(1+\frac{s a_{Y}}{10}\right)}{a_{X}\left(1-\frac{s a_{X}}{10}\right)+b_{X}\left(1-\frac{s b_{X}}{10}\right)} .
$$

Similarly, the lower limit in (A3) is then decreased to a $95 \%$ confidence minimum:

$$
L_{X}\left(\frac{a_{X}+b_{X}}{a_{Y}+b_{Y}}\right) \rightarrow \frac{a_{X}\left(1-\frac{s a_{X}}{10}\right)}{a_{Y}\left(1+\frac{s a_{Y}}{10}\right)+b_{Y}\left(1+\frac{s b_{Y}}{10}\right)} .
$$

Lastly, step 4 of Algorithm 1 limits the resulting confidence interval to $(0,1)$. Therefore the final bound for $L_{Z}$ is given by (A6). Inequality (A7) is an abbreviated version of (A6) in which $M_{L}$ and $M_{U}$ are written in place of the quotient arguments to the max and $\min$ functions.

$$
\begin{gathered}
\max \left(0, \frac{a_{X}\left(1-\frac{s a_{X}}{10}\right)}{a_{Y}\left(1+\frac{s a_{Y}}{10}\right)+b_{Y}\left(1+\frac{s b_{Y}}{10}\right)}\right)<L_{Z}<\min \left(1, \frac{a_{Y}\left(1+\frac{s a_{Y}}{10}\right)}{a_{X}\left(1-\frac{s a_{X}}{10}\right)+b_{X}\left(1-\frac{s b_{X}}{10}\right)}\right) \\
\max \left(0, M_{L}(X, Y)\right)<L_{Z}<\min \left(1, M_{U}(X, Y)\right)
\end{gathered}
$$

Note that if agents $X$ and $Y$ have closely aligned sentiments (i.e. $L_{X} \sim L_{Y}$ ), the upper limit in (A6) is marginally greater than $L_{Y}$ and the lower limit in (A6) is marginally less than $L_{X}$. The result (A6) applies generally for the interaction between any pair of agents. With each interaction the bounds are extended further. For $n$ agents $X=\left\{X_{i}\right\}_{i=1 . . n}$, and $r$ interactions, let the set $\boldsymbol{X}_{[n, r]}$ be the resultant of all those interactions. Further let $\bar{X}_{[n, r]}$ be the weighted mean (Section 3.3) location of the members of $\boldsymbol{X}_{[n, r]}$ Therefore we can write (in the notation of (A7)): 


$$
\max \left(0, \min _{r}\left(M_{L}\left(\bar{X}_{[n, r]}\right)\right)\right)<L_{Z}<\min \left(1, \max _{r}\left(M_{U}\left(\bar{X}_{[n, r]}\right)\right)\right)
$$

Equation (A8) is the desired result for a Group of agents.

\section{APPENDIX B: CONVERGENCE OF THE GROUP LOCATION}

This result is first mentioned in section 3.3, and is an extension of the result of Appendix A. Convergence of the Group Location may be summarised by the statement:

The interval in which the expected location of the resultant of an interaction between any two agents in a Group $\boldsymbol{X}$ is a subset of the corresponding interval for the previous interaction.

This result shows that the expected Group Location converges to somewhere in $(0,1)$ but does not specify where. The same notation that was used in Appendix A is used here. Let $U_{r}$ be the upper limit of the expected value of the locations of members of the Group $\boldsymbol{X}$ after $r$ interactions. Let $V_{r}$ be the corresponding lower limit. Then

$$
U_{r}=\max _{r}\left(E\left(\boldsymbol{X}_{[n, r]}\right)\right) ; V_{r}=\min _{r}\left(E\left(\boldsymbol{X}_{[n, r]}\right)\right)
$$

Let $L_{X_{i}}(r)$ be the location of agent $X_{i}$ after $r$ interactions. At interaction $r+1$, the location of $X_{i}$ changes under the influence of an agent $Y_{i}$. Using $\Phi\left(0, s^{2}\right)$ for a single draw from a Normal distribution as in Section 3.2, for some $\mu_{r} \in(0,1)$ :

$$
\begin{gathered}
L_{X_{i}}(r+1)=\mu_{r} L_{X_{i}}(r)+\left(1-\mu_{r}\right) L_{Y_{i}}(r)+\Phi\left(0, s^{2}\right) \\
\therefore E\left(L_{X_{i}}(r+1)\right)=\mu_{r} E\left(L_{X_{i}}(r)\right)+\left(1-\mu_{r}\right) E\left(L_{Y_{i}}(r)\right)+0 \\
\therefore E\left(L_{X_{i}}(r+1)\right) \in\left(V_{r}, U_{r}\right) \in(0,1) \\
\therefore\left(V_{r+1}, U_{r+1}\right) \in\left(V_{r}, U_{r}\right)
\end{gathered}
$$

Equation (B3) establishes local convergence, effectively an expected reversion to the centre of the interval $(0,1)$. The expected diminution of the interval in (B3) shows an expected progressive reduction in the variance of the location of Group $\boldsymbol{X}$ with increasing $r$. 